\title{
Self-Assembly of Luminescent Twisted Fibers Based on Achiral Quinacridone Derivatives
}

\author{
Yunfeng Zhao', Yan Fan ${ }^{1}$, Xiaoyue Mu${ }^{1}$, Hongze Gao ${ }^{1}$, Jia Wang ${ }^{1}$, Jingying Zhang ${ }^{1}$, Wensheng Yang ${ }^{1}$, Lifeng \\ $\mathrm{Chi}^{2}$, and Yue Wang ${ }^{1}(\bowtie)$ \\ ${ }^{1}$ State Key Laboratory of Supramolecular Structure and Materials, College of Chemistry, Jilin University, Changchun 130012, China \\ ${ }^{2}$ Physikalisches Institut and Center for Nanotechnology (CeNTech), Universität Münster, Münster 48149, Germany \\ Received: 12 December 2008 / Revised: 31 March 2009 / Accepted: 1 April 2009 \\ CTsinghua University Press and Springer-Verlag 2009. This article is published with open access at Springerlink.com
}

\begin{abstract}
It is a great challenge to spontaneously assemble achiral molecules into twisted nanostructures in the absence of chiral substances. Here we show that two achiral centrosymmetric quinacridone (QA) derivatives, $N, N^{\prime}$ di(n-hexyl)-1, 3, 8, 10-tetramethylquinacridone (C6TMQA) and $N, N^{\prime}$-di(n-decyl)-1, 3, 8, 10-tetramethylquinac ridone (C10TMQA), can be employed as building blocks to fabricate well-defined twisted nanostructures by controlling the solvent composition and concentration. Bowknot-like bundles with twisted fiber arms were prepared from C6TMQA, whilst uniform twisted fibers were generated from C10TMQA in ethanol/THF solution. Spectroscopic characterization and molecular simulation calculations revealed that the introduction of ethanol into the solution could induce a staggered aggregation of C6TMQA (or C10TMQA) molecules and the formation of twisted nanostructures. Such twisted materials generated from achiral organic functional molecules may be valuable in the design and fabrication of new materials for optoelectronic applications.
\end{abstract}

\section{KEYWORDS}

Twisted fiber, quinacridone, self-assembly, luminescence

The self-assembly of twisted or helical nanostructures based on functional organic molecules has become a hot topic. The construction of these nano-structures is very important in increasing our understanding of some life substances and may afford a promising approach toward the design and synthesis of materials with potential applications in areas such as sensors, liquid crystals, and optical activity [1-6]. Three strategies have been employed to construct helical or twisted structures. Generally, the supramolecular helical or twisted objects have been generated by assembling small organic molecules containing chiral groups such as peptides, lipid bilayers, glucose, bola-amphiphiles, or $\pi$-conjugant oligomers [7-12]. In some cases, achiral molecules can be assembled into twisted or helical fibers through the introduction of chiral molecule templates [13, 14]. Furthermore, some highly anisotropic achiral molecules such as amphiphilic cyanine dyes, cationic surfactants, and asymmetric porphyrins, have been used to prepare twisted or helical nano-structures [15-20]. The design and synthesis of achiral organic functional molecules which can assemble into well-defined twisted or helical architectures in the

Address correspondence to yuewang@jlu.edu.cn 
absence of chiral substances are still great challenges. Investigating this phenomenon is important in understanding the origin of chirality and may lead to efficient methods for the fabrication of chiral materials with novel properties [21, 22]. Quinacridone (QA) and its derivatives have displayed excellent photovoltaic [23, 24] and organic electroluminescent $[25,26]$ properties. The three-dimensional (3-D) supramolecular structures in crystals [27-29] and the two-dimensional (2-D) assembly properties in films [30-33] of QA derivatives have been extensively investigated. Recently, we have successfully constructed one-dimensional (1-D) nanofibers based on QA derivatives, and the size-dependent luminescence properties of the QA-based nanofibers have been documented [34]. In this contribution, we report that a dopant solvent can induce the hierarchical self-assembly of twisted structures with luminescent properties based on achiral centrosymmetric $\mathrm{QA}$ derivatives.

The QA derivatives (Scheme 1), $N, N^{\prime}$-di( $n$-hexyl)1,3,8,10-tetramethylquinacridone (C6TMQA) and $N, N^{\prime}$-di(n-decyl)-1,3,8,10-tetramethylquinacridone (C10TMQA), were synthesized according to the published procedure $[28,34]$. The solution evaporation method was used to fabricate the samples. A $50 \mu \mathrm{L}$ aliquot of C6TMQA or C10TMQA in ethanol/THF (THF = tetrahydrofuran) solution was dropped on a substrate located in a glass container with a cover. After natural evaporation of the solvents at room temperature, the samples were generated on the substrate.

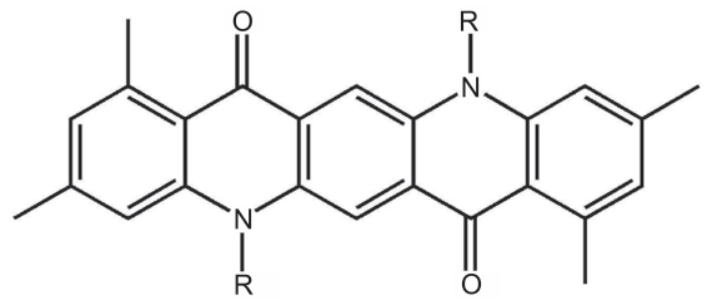

C6TMQA: $\mathrm{R}=\left(\mathrm{CH}_{2}\right)_{5} \mathrm{CH}_{3} ; \mathrm{C}$ 10TMQA: $\mathrm{R}=\left(\mathrm{CH}_{2}\right)_{9} \mathrm{CH}_{3}$

Scheme 1 Molecular structures of C6TMQA and C10TMQA

Firstly, C6TMQA molecules were used as building blocks to fabricate twisted fibers. For a C6TMQA solution with a concentration of $1.0 \times 10^{-4} \mathrm{~mol} / \mathrm{L}$, bowknot-like bundles were generated on the substrate when the molar ratio of ethanol/C6TMQA $\left(R_{\mathrm{C} 6}\right)$ was in the range 1000 to 12,000 . Fluorescence microscopy and field emission scanning electron microscopy (FESEM) revealed that the bowknot-like bundles were formed from twisted fibers (Figs. 1(a)-(c)). The arm diameters of the bowknots were about $200 \mathrm{~nm}$ with lengths of up to several micrometers. For a given concentration of C6TMQA, the $R_{\mathrm{C} 6}$ value is critical for the formation of twisted structures. Flat fibers were generated from a C6TMQA solution with a concentration of $1.0 \times 10^{-4} \mathrm{~mol} / \mathrm{L}$, when $R_{\mathrm{C} 6}$ was less than 1000. When $R_{\mathrm{C} 6}$ was in the range 12,000 to 50,000 , a mixture of the bowknot-like bundles and some diamond-like microcrystals were obtained. When $R_{\mathrm{C} 6}$ was over 50,000 for the same concentration of C6TMQA, diamond-like microcrystals with dimensions of $10 \mu \mathrm{m} \times 10 \mu \mathrm{m} \times 1 \mu \mathrm{m}$ were formed on the substrate (Fig. S-1 in the Electronic Supplementary Material (ESM)). For a more concentrated solution $\left(1.4 \times 10^{-3} \mathrm{~mol} / \mathrm{L}\right)$, when $R_{\mathrm{C} 6}$ was in the range 500 to 3000 , the bowknot-like bundles were formed together with some diamond-like microcrystals (Fig. 1(d)). Flat fibers and microcrystals were formed when $R_{\mathrm{C} 6}$ was less than 500 and the diamond-like microcrystals alone were formed when $R_{\mathrm{C} 6}$ was over

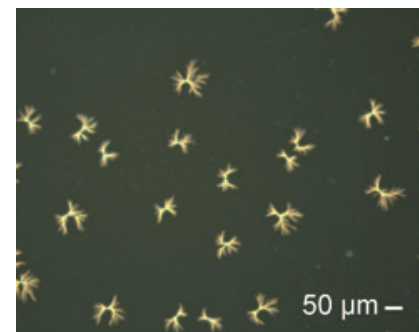

(a)

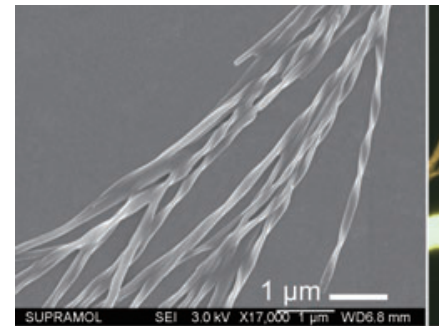

(c)

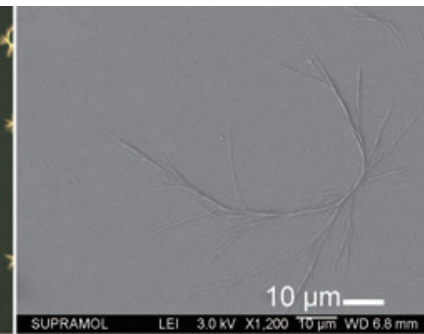

(b)

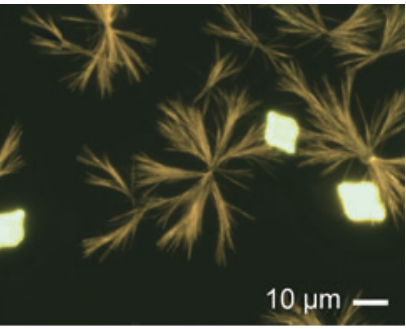

(d)
Figure 1 Morphologies of C6TMQA bowknot-like bundles prepared from dilute solution $\left(1.0 \times 10^{-4} \mathrm{~mol} / \mathrm{L}, R_{\mathrm{C} 6}=10000\right)$ : Fluorescence microscopy image on a large scale (a), FESEM image of a single bowknot (b) and its twist arms (c). Fluorescence microscopy image of bowknot-like bundles and diamond-like microcrystals prepared from a more concentrated solution $\left(1.4 \times 10^{-3} \mathrm{~mol} / \mathrm{L}, R_{\mathrm{C} 6}=500\right)(\mathrm{d})$ 
3000. The solubility of C6TMQA in ethanol is poor, while that in THF is good. Upon solvent evaporation of the C6TMQA solution in ethanol/THF, the ratio of ethanol to THF will increase because THF is more volatile than ethanol [35]. For C6TMQA solutions with a high $R_{\mathrm{C} 6}$ value, the solvent evaporation induces rapid crystallization of C6TMQA molecules and the formation of the diamond-like microcrystals [34].

In order to study the assembly of QA derivatives with different molecular structures, C10TMQA molecules with longer alkyl chains were employed. When a C10TMQA THF solution with a concentration of $1.0 \times 10^{-4} \mathrm{~mol} / \mathrm{L}$ was used, twisted fibers were obtained when the molar ratio of ethanol/C10TMQA $\left(R_{\mathrm{C} 10}\right)$ ranged from 6400 to 15,000 . The twisted fibers exhibited orange emission as recorded by fluorescence microscopy (Fig. 2(a)). FESEM microscopy images illustrate that the twisted fibers are formed by coiling of thinner fibers (Figs. 2(b)-(d)) and that the diameter of the twisted fibers was about $300 \mathrm{~nm}$ and the twist pitch was about $500 \mathrm{~nm}$, based on the statistical evaluation of about 1000 pieces of twisted fibers. For the same concentration of C10TMQA, when $R_{\mathrm{C} 10}$ was less than 3400, flat fibers similar to those prepared from pure THF solution [34] were obtained (Fig. S-2(a) in the SEM). When $R_{\mathrm{C} 10}$ was between 3400 and 6400 , the twisted and flat fibers were generated, simultaneously (Figs. S-2(b) and (c) in the ESM). The twisted fibers became more

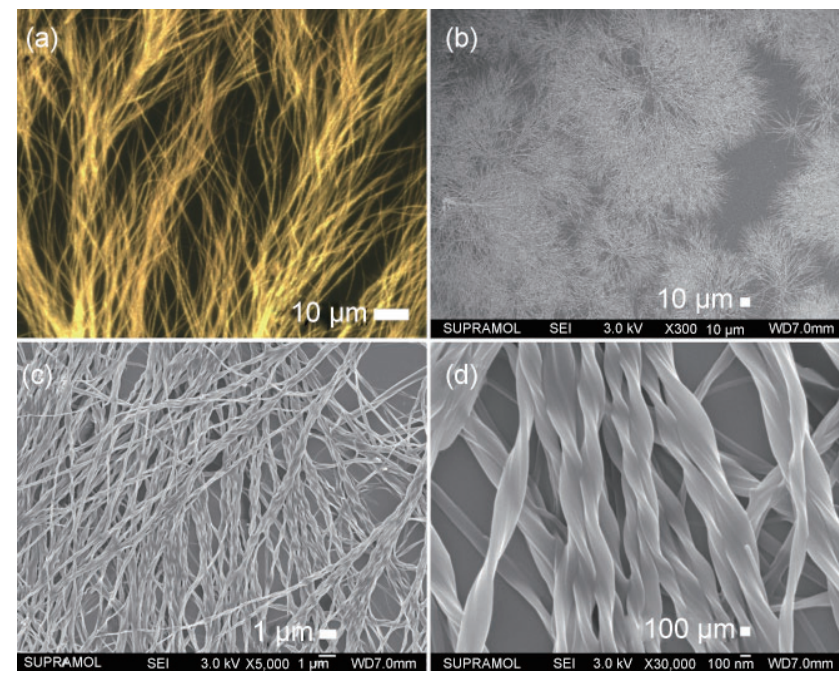

Figure 2 Fluorescence microscopy image (a) and FESEM images (b)(d) of twisted fibers prepared from dilute $\left(1.0 \times 10^{-4} \mathrm{~mol} / \mathrm{L}\right) \mathrm{C}$ 10TMQA THF solution doped with ethanol $\left(R_{\mathrm{c} 10}=15,000\right)$ curled when $R_{\mathrm{C} 10}$ was over 15,000 for the solution with concentration of $1.0 \times 10^{-4} \mathrm{~mol} / \mathrm{L}$ (Fig. S-2(d) in the ESM).

The morphologies of the twisted structures are concentration-dependent. For a more concentrated solution $\left(3.0 \times 10^{-3} \mathrm{~mol} / \mathrm{L}\right)$, more diversified twisted structures were generated from C10TMQA solutions with $R_{\mathrm{C} 10}$ values from 500 to 3500 . The twisted fibers displayed orange luminescence and self-wave-guided emission characteristics, which could be observed by luminescence microscope imaging (Fig. 3(a)). The FESEM images further illustrate that a variety of hierarchical self-assembled structures such as single twisted fibers, single twisted tapes, twisted bundles, and twisted tubes were formed (Figs. 3(b)-(d)). The different morphologies had different twist pitches of $2.05 \mu \mathrm{m}$ for single twisted fibers, $2.52 \mu \mathrm{m}$ for single twisted tapes, $5.35 \mu \mathrm{m}$ for bundled twisted fibers, and $6.4 \mu \mathrm{m}$ for twisted tubes. It is worth noting that the twisted tube is enclosed by four twisted tapes (Fig. 3(d)). The C10TMQA molecules partly selfassembled into twisted fibers if $R_{\mathrm{C} 10}$ was less than 500 (Fig. S-3 in the ESM). When $R_{\mathrm{C} 10}$ was over 3500 , the twisted fibers were more curled. Owing to the lack of chirality in C6TMQA, C10TMQA, and the solvents, both left- and right-handed twisted fibers were observed in all cases, and the twisted samples were racemic mixtures. The twisted morphologies could be prepared on different substrates (silica, glass, mica,
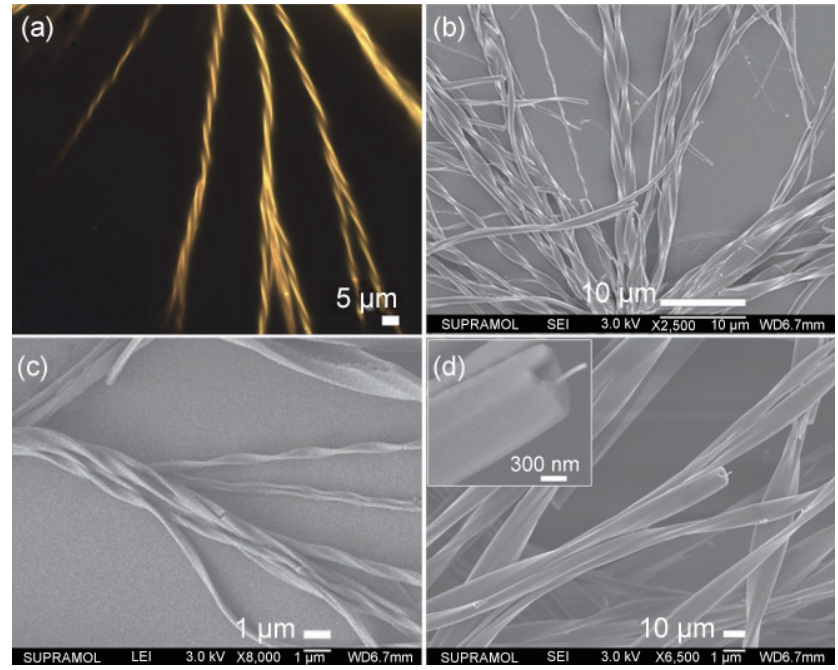

Figure 3 Luminescence microscopy (a) and FESEM images (b)-(d) of nano-twisted structures prepared from higher concentration (3.0x $\left.10^{-3} \mathrm{~mol} / \mathrm{L}\right)$ C 10TMQA THF solution doped with ethanol $\left(R_{\mathrm{C} 10}=700\right)$ 
aluminium foil and indium tin oxide (ITO) glass) (Fig. S-4 in the ESM) suggesting that these twisted fibers may be used to fabricate microelectric devices on such substrates.

In addition, some other solvents, such as water, aliphatic alcohols (methanol, $n$-propanol, $i$-propanol, $n$-butanol, t-butanol, n-pentanol, $n$-hexanol, $n$-heptanol, $n$-octanol, and $n$-decanol) and aliphatic acids (formic acid, acetic acid, propionic acid, and butyric acid), were also used as dopant solvents to study the assembly properties of C10TMQA in different mixed solutions. However, the twisted fibers were generated only in $n$-propanol/THF, $i$-propanol/ THF, acetic acid/THF, and propionic acid/THF solution systems $\left(3.0 \times 10^{-3} \mathrm{~mol} / \mathrm{L}, R_{\mathrm{C} 10}=700\right)$ (Fig. $\mathrm{S}-5$ in the ESM). The twisted fibers could also be generated from dilute solutions of C10TMQA in pure ethanol, $n$-propanol or $i$-propanol (Fig. S-6 in the ESM). These results illustrate that the molecular structures and polarity of the dopant solvents have a critical effect on the twisted self-assembly properties of C10TMQA molecules.

Figure 4 displays the normalized absorption spectra recorded for THF solutions od C10TMQA with a constant concentration $\left(1.0 \times 10^{-4} \mathrm{~mol} / \mathrm{L}\right)$ and different $R_{\mathrm{C} 10}$ values. The electronic absorption of C10TMQA exhibits an obvious coupling to the vibronic features corresponding to the $v=0$ to $v^{\prime}=0,1$, and 2 transitions. Upon increasing $R_{\mathrm{C} 10}$, the absorption spectra exhibit a gradual and obvious

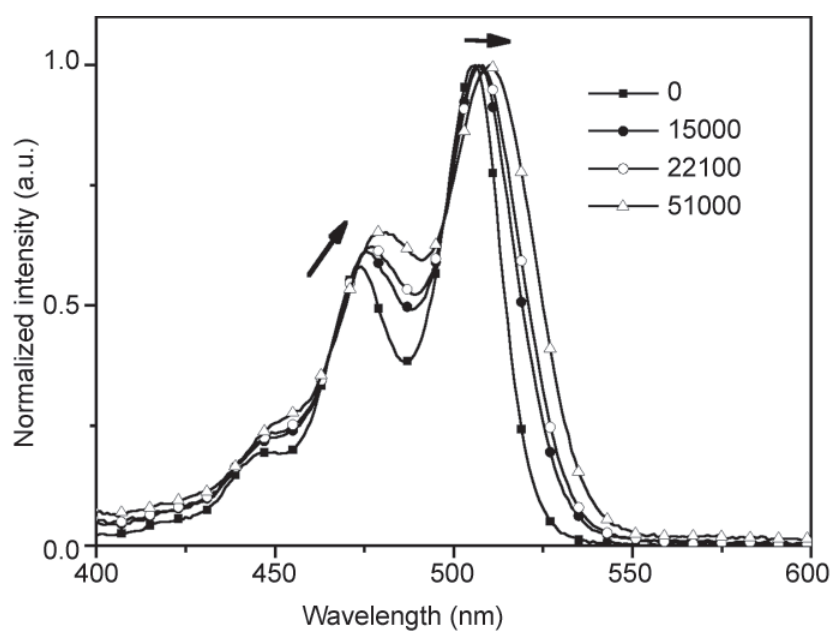

Figure 4 Normalized ethanol-dependent UV-Vis absorption spectra of C10TMQA $\left(1.0 \times 10^{-4} \mathrm{~mol} / \mathrm{L}\right)$ for different values of $R_{\mathrm{C} 10}$ ranging from 0 to 51,000 increase of the $A^{0 \rightarrow 1} / A^{0 \rightarrow 0}$ value (the ratio of the absorbance of the $0 \rightarrow 1$ transition to that of $0 \rightarrow 0$ ) accompanied by a slight red-shift. For THF solutions of C10TMQA with higher concentration $\left(1.3 \times 10^{-3}\right.$ mol/L), similar $R_{\mathrm{C} 10}$-dependent absorption properties were also observed (Fig. S-7 in the ESM). In addition, when $R_{\mathrm{C} 10}$ reached 19,600 for the more concentrated solution, some twisted fibers were observed in the solution system. Experimental and theoretical studies have revealed that the typical face-to-face aggregation or $\mathrm{H}$-aggregation of aromatic $\pi$-systems can result in an increase in the intensities of $0 \rightarrow 1$ and $0 \rightarrow 2$ transitions, and in turn lead to an increase of the $A^{0 \rightarrow 1} / A^{0 \rightarrow 0}$ value $[36,37]$, while the slight red-shift of the absorption spectra may be attributed the increase in particle size [38].

To understand the molecular packing mode of the twisted structures, several measurements were performed on twisted and flat fiber samples. Differential scanning calorimetry (DSC) showed that the twisted and flat fibers have different thermodynamic behaviors (Fig. S-8(a) in the ESM). The flat fiber displayed only a melting peak at around $182{ }^{\circ} \mathrm{C}$, whereas the twisted samples prepared from different doped alcohols (ethanol, n-propanol, and $i$-propanol) all exhibited a small endothermic peak at about $100{ }^{\circ} \mathrm{C}$, and then melted at about $182{ }^{\circ} \mathrm{C}$. Thermogravimetric analysis (TGA) curves showed that the twisted fibers lost $3.20 \%$ in weight between $70{ }^{\circ} \mathrm{C}$ and $130^{\circ} \mathrm{C}$, which can be attibuted to the loss of small molecules (Fig. S-8(b) in the ESM). The twisted and flat fibers showed quite different powder X-ray diffraction (XRD) patterns, while the annealed twisted fibers displayed similar an XRD pattern to that of the flat fibers (Fig. S-9 in the ESM). Although the twisted fibers heated at below $110{ }^{\circ} \mathrm{C}$ still retained a twisted morphology, they displayed a green fluorescence, similar to that shown by the flat fibers (Fig. 5). These results suggest that the twisted samples may undergo a solid-state phase transformation process because of loss of ethanol upon annealing [39], and that the twisted and flat fibers had different molecular packing properties with the molecular packing mode of the twisted fibers being transformed into that of the flat fibers upon heating. The XRD pattern (Fig. S-9 in the ESM) calculated from the single crystal X-ray 

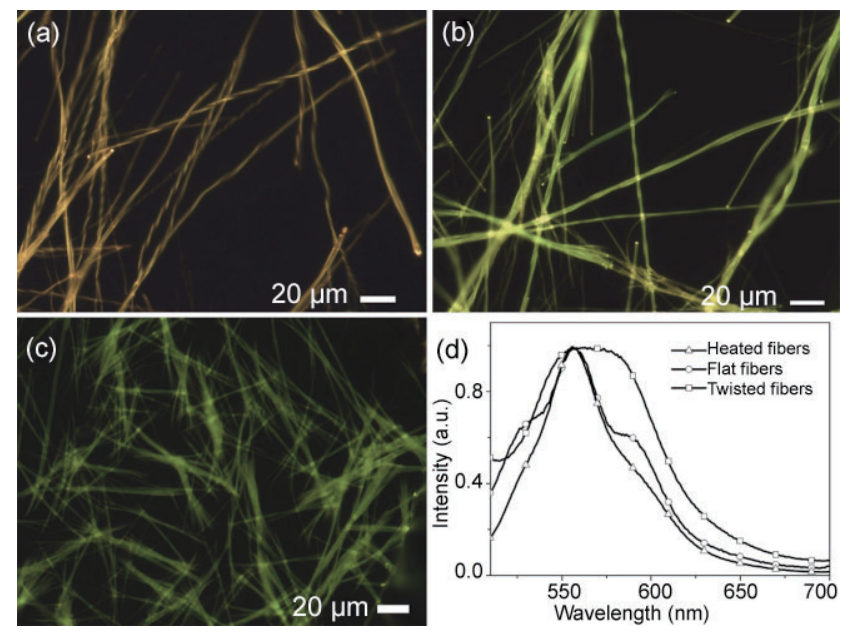

Figure 5 Fluorescence images of C10TMQA twisted fibers (a), annealed twisted fibers (b), and flat fibers (c). Fluorescence spectra $\left(\lambda_{\mathrm{ex}}=470 \mathrm{~nm}\right)$ of twisted fibers, flat fibers and heated twisted fibers (d)

data of C10TMQA is similar to that for the flat fibers, suggesting that the molecular packing structure of the flat fibers is analogous to that in single crystals of C10TMQA.

A single crystal X-ray diffraction study (the details are given in the ESM) revealed that the molecular packing structure of the C10TMQA crystal displays obviously anisotropic features. Along the $b$ axis direction the C10TMQA molecules stack into infinite 1-D molecular columns (Fig. 6(a)). The crystal structure suggests that C10TMQA molecules have a natural tendency to aggregate into linear 1-D structures. The single crystal structure of C10TMQA therefore provides a rational explanation for the formation of the flat fibers. To understand the mechanism of formation of the twisted structures, molecular simulations were performed on the C10TMQA molecule with and without ethanol. First, the geometries of the C10TMQA monomer without any ethanol and the C10TMQA monomer with two ethanol molecules were optimized at the B3LYP/3-21G level. Then, the C10TMQA monomers with or without ethanol were arranged in parallel to form dimers. Finally, geometry optimizations of the C10TMQA dimers with or without ethanol molecules were carried out based on the Gaussian 03 implementation of semiempirical AM1 calculations [40]. The calculated results show that for the C10TMQA dimer without ethanol molecules the two
C10TMQA molecules adopt a parallel orientation (Fig. 6(b)), which is similar to the structure in the single crystal. However, for the C10TMQA dimer with four ethanol molecules, the two C10TMQA molecules display a staggered orientation (Fig. 6(c)). Figures 6(c)-L and 6(c)-R display the mirror image features of the left and right handed C10TMQA dimers, confirming the chiral characteristic of the dimers with ethanol molecules. If the staggered C10TMQA dimers (with four ethanol molecules) are arrayed along the calculated orientation, a twisted structure can be generated (Fig. 6(d)). The actual mechanism of formation of the twisted structures reported in this study is likely to be very complicated, and therefore, the above calculations can only demonstrate that in the presence of the ethanol molecules, C10TMQA molecules have the tendency to aggregate into the twisted structure. At present, we cannot give any further explanation of this phenomenon. In fact, FTIR and ${ }^{1} \mathrm{H}$ NMR spectroscopy showed that ethanol molecules really are co-assembled with C10TMQA molecules (Figs. S-10 and S-11 in the ESM) confirming that ethanol molecules are essential for the formation of twisted structures.

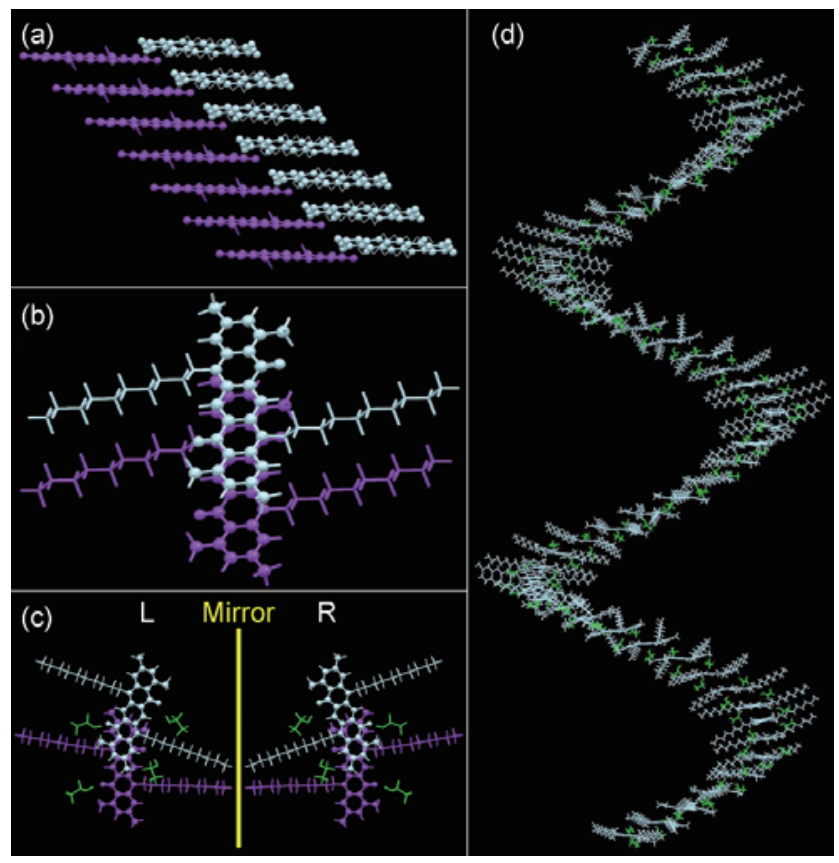

Figure 6 (a) Column packing in a single crystal of C10TMQA. Molecular simulations of C10TMQA molecules packing without ethanol (b) and with ethanol (c) using B3LYP/3-21G and AM1, and the twisted structure (d) expanded from 28 twisted dimers 
In conclusion, the achiral C6TMQA and C10TMQA molecules are able to spontaneously assemble into twisted nanostructures in their solutions in THF with certain concentrations and co-solvent compositions. The introduction of ethanol into the solution induces the staggered aggregation of C6TMQA or C10TMQA molecules and the formation of twisted structures upon the evaporation of THF and ethanol. For a THF solution of C6TMQA or C10TMQA, the molar ratio of ethanol/C6TMQA or C10TMQA (namely $R_{\mathrm{C} 6}$ or $R_{\mathrm{C} 10}$ ) has a dramatic effect on the assembled structures. When $R_{\mathrm{C} 6}$ or $R_{\mathrm{C} 10}$ was controlled within a particular range, twisted structures could be easily obtained. These twisted materials generated from achiral organic functional molecules may be valuable in the design and fabrication of new materials for optoelectronic applications.

\section{Acknowledgements}

This work was supported by the National Natural Science Foundation of China (50733002 and 50773027), the Major State Basic Research Development Program (2009CB939700) and 111 Project (B06009).

Electronic Supplementary Material: Supplementary Material (11 figures and details of single crystal X-ray structure determination) is available in the online version of this article at http://dx.doi.org/10.1007/ s12274-009-9045-4 and is accessible free of charge.

\section{References}

[1] Engelkamp, H.; Middelbeek, S.; Nolte, R. J. M. Selfassembly of disk-shaped molecules to coiled-coil aggregates with tunable helicity. Science 1999, 284, 785 -788 .

[2] Mateos-Timoneda, M. A.; Crego-Calama, M.; Reinhoudt, D. N. Supramolecular chirality of self-assembled systems in solution. Chem. Soc. Rev. 2004, 33, 363-372.

[3] van der Jonkheijm, P.; Schoot, P.; J. Schenning, A. P. H.; Meijer, E. W. Probing the solvent-assisted nucleation pathway in chemical self-assembly. Science 2006, 313, 80-83.

[4] Amabilino, D. B.; Veciana, J. Supramolecular chiral functional materials. Top. Curr. Chem. 2006, 265, 252302.

[5] Palmer, L. C.; Velichko, Y. S.; de la Cruz, M. O.; Stupp, S. I. Supramolecular self-assembly codes for functional structures. Phil. Trans. R. Soc. A 2007, 365, 1417-1433.

[6] Niu, Z.; Bruckman, M. A.; Harp, B.; Mello, C. M.; Wang, Q. Bacteriophage M13 as a scaffold for preparing conductive polymeric composite fibers. Nano Res. 2008, 1, 235-241.

[7] Fuhrhopa, J. -H.; Helfrich, W. Fluid and solid fibers made of lipid molecular bilayers. Chem. Rev. 1993, 93, 15651582.

[8] Thalacker, C.; Würther, F. Chiral perylene bisimidemelamine assemblies: Hydrogen bond-directed growth of helically stacked dyes with chiroptical properties. Adv. Funct. Mater. 2002, 12, 209-218.

[9] Brizard, A.; Oda, R.; Huc, I. Chirality effects in selfassembled fibrillar networks. Top. Curr. Chem. 2005, 256, 167-218.

[10] Bae, J.; Choi, J. -H.; Yoo, Y. -S.; Oh, N. -K.; Kim, B. -S.; Lee, M. Helical nanofibers from aqueous self-assembly of an oligo(p-phenylene)-based molecular dumbbell. J. Am. Chem. Soc. 2005, 127, 9668-9669.

[11] Ajayaghosh, A.; Praveen, V. K. pi-Organogels of selfassembled $p$-phenylenevinylenes: Soft materials with distinct size, shape, and functions. Acc. Chem. Res. 2007, 40, 644-656.

[12] Goh, M.; Kyotani, M.; Akagi, K. Highly twisted helical polyacetylene with morphology free from the bundle of fibrils synthesized in chiral nematic liquid crystal reaction field. J. Am. Chem. Soc. 2007, 129, 8519-8527.

[13] Maeda, K.; Yashima, E. Dynamic helical structures: Detection and amplification of chirality. Top. Curr. Chem. 2006, 265, 47-88.

[14] Palmans, A. R. A.; Meijer, E. W. Amplification of chirality in dynamic supramolecular aggregates. Angew. Chem. Int. Ed. 2007, 46, 8948-8968.

[15] Fejer, S. N.; Wales, D. J. Helix self-assembly from anisotropic molecules. Phys. Rev. Lett. 2007, 99, 086106.

[16] Yang, W.; Chai, X.; Chi, L.; Liu, X.; Cao, Y.; Lu, R.; Jiang, Y.; Tang, X.; Fuchs, H.; Li, T. From achiral molecular components to chiral supermolecules and supercoil selfassembly. Chem, Eur. J. 1999, 5, 1144-1149.

[17] Takeuchi, M.; Tanaka, S.; Shinkai, S. On the influence of porphyrin $\pi-\pi$ stacking on supramolecular chirality created in the porphyrin-based twisted tape structure. 
Chem. Commun. 2005, 5539-5541.

[18] Yuan, J.; Liu, M. Chiral molecular assemblies from a novel achiral amphiphilic 2-(heptadecyl)naphtha[2,3]im idazole through interfacial coordination. J. Am. Chem. Soc. 2003, 125, 5051-5056.

[19] Huang, X.; Li, C.; Jiang, S.; Wang, X.; Zhang, B.; Liu, M. Self-assembled spiral nanoarchitecture and supramolecular chirality in Langmuir-Blodgett films of an achiral amphiphilic barbituric acid. J. Am. Chem. Soc.

2004, 126, 1322-1323.

[20] Simon, F. -X.; Khelfallah, N. S. Formation of helical mesopores in organic polymer matrices. J. Am. Chem. Soc. 2007, 129, 3788-3789.

[21] Sly, J.; Kasák, P.; Gomar-Nadal, E.; Rovira, C.; Górriz, L.; Thordarson, P.; Amabilino, D. B.; Rowan, A. E.; Nolte, R. J. M. Chiral molecular tapes from novel tetra(thiafulvalenecrown-ether)-substituted phthalocyanine building blocks. Chem. Commun. 2005, 1255-1257.

[22] Wang, M.; Yang, Y. -L.; Deng, K.; Wang, C. Uncoiling process of helical molecular fibrillar structures studied by AFM. J. Phys. Chem. C 2007, 111, 6194-6198.

[23] Hiramoto, M.; Kawase, S.; Yokoyama, M. Photoinduced hole injection multiplication in p-type quinacridone pigment films. Jpn. J. Appl. Phys. 1996, 35, L349-L351.

[24] Shichiri, T.; Suezaki, M.; Inoue, T. Three-layer organic solar cell. Chem. Lett. 1992, 21, 1717-1720.

[25] Shi, J.; Tang, C. W. Doped organic electroluminescent devices with improved stability. Appl. Phys. Lett. 1997, 70, 1665-1667.

[26] Gross, E. M.; Anderson, J. D.; Slaterbeck, A. F.; Thayumanavan, S.; Barlow, S.; Zhang, Y.; Marder, S. R.; Hall, H. K.; Nabor, M. F.; Wang, J. F. et al. Electrogenerated chemiluminescence from derivatives of aluminum quinolate and quinacridones: Crossreactions with triarylamines lead to singlet emission through triplet-triplet annihilation pathways. J. Am. Chem. Soc. 2000, 122, 4972-4970.

[27] Lincke, G. A review of thirty years of research on quinacridone. X-ray crystallography and crystal engineering. Dyes Pigm. 2000, 44, 101-122.

[28] Ye, K. Q.; Wang, J.; Sun, H.; Liu, Y.; Mu, Z. C.; Li, F.; Jiang, S. M.; Zhang, J. Y.; Zhang, H. X.; Wang, Y. et al. Supramolecular structures and assembly and luminescent properties of quinacridone derivatives. J. Phys. Chem. B 2005, 109, 8008-8016.
[29] Paulus, E. F.; Leusen, F. J. J.; Schmidt, M. U. Crystal structures of quinacridones. CrystEngComm, 2007, 9, 131-143.

[30] Keller, U.; Müllen, K.; De Feyter, S.; De Schryver, F. C. Hydrogen-bonding and phase-forming behavior of a soluble quinacridone. Adv. Mater. 1996, 8, 490-493.

[31] Qiu, D. L.; Ye, K. Q.; Wang, Y.; Zou, B.; Zhang, X.; Lei, S. B.; Wan, L. J. In situ scanning tunneling microscopic investigation of the two-dimensional ordering of different alkyl chain-substituted quinacridone derivatives at highly oriented pyrolytic graphite/solution interface. Langmuir 2003, 19, 678-681.

[32] Lin, F.; Zhong, D. Y.; Chi, L. F.; Ye, K. Q.; Wang, Y.; Fuchs, $\mathrm{H}$. Temperature-tuned organic monolayer growth: $\mathrm{N}, \mathrm{N}$ di(n-butyl)quinacridone on Ag(110). Phys. Rev. B 2006, 73, 235420.

[33] Trixler, F.; Markert, T.; Lackinger, M.; Jamitzky, F.; Heckl, W. M. Supramolecular self-assembly initiated by solid-solid wetting. Chem. Eur. J. 2007, 13, 7785-7790.

[34] Wang, J.; Zhao, Y. F.; Zhang, J. H.; Zhang, J. Y.; Yang, B.; Wang, Y.; Zhang, D. K.; You, H.; Ma, D. G. Assembly of one-dimensional organic luminescent nanowires based on quinacridone derivatives. J. Phys. Chem. C 2007, 111, 9177-9183.

[35] Smallwood, I. M. Handbook of Organic Solvent Properties; Arnold: London, 1996; p. xvii, p. 65, and p. 217.

[36] Wang, W.; Han, J. J.; Wang, L. Q.; Li, L. S.; Shaw, W. J.; Li, A. D. Q. Dynamic $\pi-\pi$ stacked molecular assemblies emit from green to red colors. Nano Lett. 2003, 3, 455458.

[37] Cornil, J.; dos Santos, D. A.; Beljonne, D.; Shuai, Z.; Brédas, J. L. In Semiconducting Polymers; Hadziioannou, G.; van Hutten, P. F., Eds.; Wiley-VCH: Weinheim, 2000; pp. 88-114.

[38] Horn, D.; Gieger, J. Organic nanoparticles in the aqueous phase-Theory, experiment, and use. Angew. Chem. Int. Ed. 2001, 40, 4330-4361.

[39] Hilfiker, R. Polymorphism in the Pharmaceutical Industry; Wiley-VCH: Weinheim, 2006; p.47.

[40] Frisch, M. J.; Trucks, G. W.; Schlegel, H. B.; Scuseria, G. E.; Robb, M. A.; Cheeseman, J. R.; Montgomery, J. A. Jr.; Vreven, T.; Kudin, K. N.; Burant, J. C. et al. Gaussian 03, Revision C.02. Gaussian, Inc.: Pittsburgh, PA, 2003. 